\title{
Prediction of superconductivity of $3 d$ transition-metal based antiperovskites via magnetic phase diagram
}

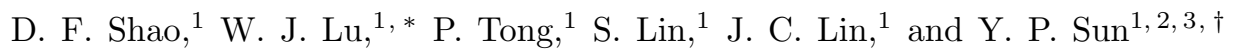 \\ ${ }^{1}$ Key Laboratory of Materials Physics, Institute of Solid State Physics, \\ Chinese Academy of Sciences, Hefei 230031, P. R. China \\ ${ }^{2}$ High Magnetic Field Laboratory, Chinese Academy of Sciences, Hefei 230031, P. R. China \\ ${ }^{3}$ University of Science and Technology of China, Hefei 230026, P. R. China
}

\begin{abstract}
We theoretically studied the electronic structure, magnetic properties, and lattice dynamics of a series of $3 d$ transition-metal antiperovskite compounds $\mathrm{AXM}_{3}$ by density function theory. Based on the Stoner criterion, we drew the magnetic phase diagram of carbon-based antiperovskites $\mathrm{ACM}_{3}$. In the phase diagram, compounds with non-magnetic ground state but locating near the ferromagnetic boundary are suggested to yield sizeable electron-phonon coupling and behave superconductivity. To approve this deduction, we systematically calculated the phonon spectra and electron-phonon coupling of a series of $\mathrm{Cr}$-based antiperovskites $\mathrm{ACCr}_{3}$ and $\mathrm{ANCr}_{3}$. The results show that $\mathrm{AlCCr}_{3}$, $\mathrm{GaCCr}_{3}$, and $\mathrm{ZnNCr}_{3}$ could be moderate coupling BCS superconductors. The influence of spin fluctuation on superconductivity are discussed. Furthermore, other potential superconducting $\mathrm{AXM}_{3}$ including some new Co-base and Fe-based antiperovskite superconductors are predicted from the magnetic phase diagram.
\end{abstract}

PACS numbers: 74.70.Ad, 74.25.Ha, 74.25.Kc, 74.20.Pq

\section{INTRODUCTION}

Since superconductivity (SC) was found by Onnes $\frac{1}{}$, researchers have made a lot of efforts to figure out its mechanism. The BCS theory $\underline{2}^{2-4}$ pointed out two electrons with opposite spins can pair with each other via electron-phonon coupling (EPC). Therefore, magnetism or spin fluctuation (SF) may break the pair and be harmful to SC. The discovery of unconventional superconductors such as cuprate oxide superconductors ${ }^{5.6}$, iron based superconductors ${ }^{7}, \frac{.8}{}, \mathrm{Sr}_{2} \mathrm{RuO}_{4}{ }^{9}, \mathrm{Na}_{x} \mathrm{CoO}_{2} \cdot y \mathrm{H}_{2} \mathrm{O}^{10}$, etc., challenges the EPC mechanism. For the unconventional superconductors, SF may play an important role in the superconducting mechanism, which makes researchers reconsider the relation between magnetism and SC.

Superconducting $\mathrm{MgCNi}_{3} \frac{11}{}$, containing a high concentration of magnetic element $\mathrm{Ni}$, attracted a lot of attentions on the role of $\mathrm{SF} . \mathrm{MgCNi}_{3}$ has a so-called antiperovskite structure, in which $\mathrm{Mg}$ atom locates at the corner, and six $\mathrm{Ni}$ atoms at the face-center together with one $\mathrm{C}$ atom at the body center form the $\mathrm{C}_{-} \mathrm{Ni}_{6}$ octahedron. A van Hove singularity locates just below Fermi level $\left(E_{F}\right)$, leading to large density of states (DOS) at $E_{F}\left(N\left(E_{F}\right) \stackrel{12-14}{\underline{14}}\right.$. It makes the compound strongly exchange-enhanced and unstable toward to ferromagnetism (FM) upon hole doping12,13. Experimentally speaking, due to the high volatility of $\mathrm{Mg}$ and the relatively poor reactivity of $\mathrm{C}$, it is extremely difficult to synthesize stoichiometric $\mathrm{MgCNi}_{3}{ }^{15-17}$. The atomic deficiencies in the antiperovskite system may strongly affect the properties $\frac{18,19}{19}$ and lead to some contradictory results in the reported polycrystalline samples $\frac{15}{}$. Recently, the single crystal samples have been prepared $16,17,20-24$. The physical property measurements demonstrate the conventional EPC mechanism, but the reported EPC strengths of these samples are contradicted $17,20-22,25,26$.
Moreover, it has not been successful so far to induce FM instability by preparing hole-doped compounds such as $\mathrm{Mg}_{1-x} \mathrm{Na}_{x} \mathrm{CNi}_{3}$ and $\mathrm{Mg}_{1-x} \mathrm{Li}_{x} \mathrm{CNi}_{3}$. The interplay between $\mathrm{SC}$ and $\mathrm{SF}$ in $\mathrm{MgCNi}_{3}$ is still unclear and in debate 27 .

Nowadays, lots of $3 d$ transition-metal based antiperovskite $\mathrm{AXM}_{3}$ (A usually is main group element; $\mathrm{X}=$ $\mathrm{B}, \mathrm{C}$, and $\mathrm{N} ; \mathrm{M}=3 d$ transition-metal elements) have been experimentally synthesized 28 . However, the reported physical property measurements are mainly focused on the $\mathrm{Ni}$ - and Mn-based antiperovskites. In Ni-based antiperovskites, $\mathrm{CdCNi}_{3}$ and $\mathrm{ZnNNi}_{3}$ were reported to show $\mathrm{SC}$ behavior ${ }^{29.30}$. Abundant magnetism appears in Mn-based antiperovskites (see recent review article Ref. 31). Moreover, superconducting trace has been found in $\mathrm{InBSc}_{3}{ }^{32}$. Some theoretical predictions suggest that SC may exist in some Sc-based and Cr-based antiperovskites ${ }^{33-36}$. Indeed, due to the high concentration of $3 d$ transition-metal atoms, one can imagine that more interesting properties exist in other antiperovskite $\mathrm{AXM}_{3}$. The investigation of them will be very important, both in the search for new superconductors and in the pursuit of a better understanding of the interplay between SC and magnetism 27 .

In the present work, we offer an approach to explore new $3 d$ transition-metal based antiperovskite superconductors. In order to have an overall understanding of the magnetism in $\mathrm{AXM}_{3}$, we calculated the electronic structures of a series of $3 d$ transition-metal based antiperovskites $\mathrm{AXM}_{3}$ and concluded the doping effects of each atom. From the analysis of these doping effects, we can evaluate the variation of $N\left(E_{F}\right)$ of different $\mathrm{AXM}_{3}$. We drew a magnetic phase diagram of $\mathrm{ACM}_{3}$ based on the Stoner criterion $N\left(E_{F}\right) I>1$. From the phase diagram, we predict that the materials locating near FM boundary may have sizeable EPC and could show SC. To con- 
firm this deduction, we systematically investigated the formation energies, electronic structures, lattice dynamic properties, and EPC of a series of Cr-based $\mathrm{AXCr}_{3}$. For comparison, such properties of $\mathrm{MgCNi}_{3}$ are also calculated. Our results confirm the strong $\mathrm{EPC}$ for $\mathrm{MgCNi}_{3}$ and suggest that $\mathrm{AlCCr}_{3}, \mathrm{GaCCr}_{3}$, and $\mathrm{ZnNCr}_{3}$ are moderate coupling BCS superconductors. The depairing effects from SF are aslo discussed. Furthermore, some potential superconducting antiperovskites such as new Coand Fe-based superconductors are suggested.

\section{METHODS}

The structural relaxation and electronic structure calculations were performed using projected augmentedwave (PAW) ${ }^{37}, 38$ method as implemented in the ABINIT code $\underline{39}-41$. Electronic wavefunctions are expanded with plane waves up to an energy cutoff $\left(E_{c u t}\right)$ of $1200 \mathrm{eV}$. Brillouin zone sampling is performed on a MonkhorstPack (MP) mesh $\frac{42}{2}$ of $16 \times 16 \times 16$. The self-consistent calculations were considered to be converged when the total energy of the system was stable within $10^{-6}$ Ha. Non-magnetic (NM) and FM states were tested in the study.

The calculations of phonon spectra and EPC were based on the frame work of the self consistent density functional perturbation theory (DFPT) ${ }^{43}$ using planewaves and ultrasoft pseudopotentials 44 with QUANTUM-ESPRESSO ${ }^{45}$. We use an $8 \times 8 \times 8$ grid for zone integration in the self-consistent calculations, while a denser $16 \times 16 \times 16$ grid was used in the EPC calculations. We have calculated dynamical matrices at a uniform $4 \times 4 \times 4$ grid of $q$-points.

To ensure our calculation reliability, we cross-checked the results given by the above two DFT codes and found them to be in close agreement. And for consistency, we used the same GGA-PBE $\underline{46}$ exchange-correlation potential in both cases.

\section{RESULTS AND DISCUSSIONS}

\section{A. Doping effects of $\mathrm{AXM}_{3}$}

As Rosner et al ${ }^{12}$ mentioned, the A-site atom only plays the role as an effective valent electrons supplier. The most common A-site atoms of the antiperoveskite compounds can be divided into four groups according to the effective valence electrons: $\mathrm{Cu}$ and $\mathrm{Ag}\left(\mathrm{A}^{1+}\right) ; \mathrm{Mg}, \mathrm{Zn}$, and $\mathrm{Cd}\left(\mathrm{A}^{2+}\right)$; $\mathrm{Al}, \mathrm{Ga}$, and $\mathrm{In}\left(\mathrm{A}^{3+}\right)$; $\mathrm{Ge}$ and $\mathrm{Sn}\left(\mathrm{A}^{4+}\right)$. Clearly, the effective valence electrons of A-site atoms are usually in the range from 1 to 4 . For simplification, we chose $\mathrm{Na}^{1+}, \mathrm{Mg}^{2+}, \mathrm{Al}^{3+}$, and $\mathrm{Si}^{4+}$ atoms as the A-site atoms of the antiperovskites we investigated.

Figure 1 shows the lattice parameters and magnetic moment of a series of carbon-based $\mathrm{ACM}_{3}$. As expected,

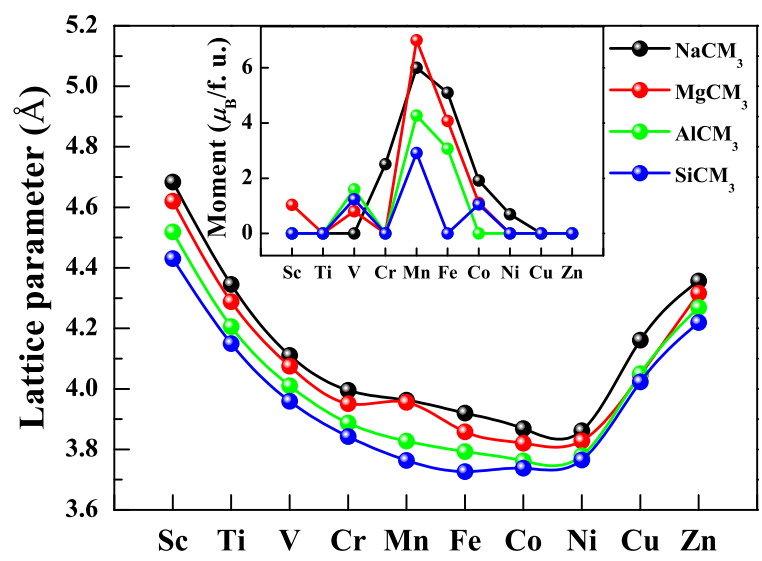

FIG. 1. Lattice parameters and magnetic moments of $\mathrm{ACM}_{3}$ $(\mathrm{A}=\mathrm{Na}, \mathrm{Mg}, \mathrm{Al}$, and $\mathrm{Si}, \mathrm{M}=3 d$ transition metal elements $)$.

the lattice parameter varies with the same trend of variation of the atom radius. The ground states of these compounds are determined by comparing the total energies of the $\mathrm{NM}$ and FM states. The $\mathrm{ACMn}_{3}$ and most $\mathrm{ACFe}_{3}$ show FM state, which coincides with the experimental results. Surprisingly, $\mathrm{SiCFe}_{3}$ and $\mathrm{AlCCo}_{3}$ show NM state. Comparing the magnetic moments with the $N\left(E_{F}\right)$ (table【), one can see that all the compounds with FM ground state have quite large $N\left(E_{F}\right)$ in NM state. It is corresponding to the Stoner's itinerant magnetism: the higher $N\left(E_{F}\right)$ in NM state, the easier a system becomes spin-polarized. The magnetism of these compounds will be discussed below.

In order to have an overall understanding of the $3 d$ transition-metal based antiperovskites $\mathrm{AXM}_{3}$, the doping/substitution effects of each atom on the electronic structure must be investigated. We start from reviewing the electronic structure of $\mathrm{MgCNi}_{3}$. Figure 2 shows the calculated DOS of $\mathrm{MgCNi}_{3}$. It can be seen that the total DOS near $E_{F}$ are mainly contributed by Ni-3d electrons. From $-7 \mathrm{eV}$ to $-4 \mathrm{eV}$ and near $E_{F}$, C- $2 p$ electrons hybridize strongly with Ni-3d electrons. The $\pi^{*}$ antibonding state locates just below $E_{F}$, which leads to the van Hove singularity, yielding the high $N\left(E_{F}\right)=2.57$ states $/ \mathrm{eV} / \mathrm{spin}$. We calculated the Stoner parameter $I$ using the methods mentioned by Rosner et al .12 . The result shows that $I=0.28$, and Stoner enhancement factor $S=\left(1-N\left(E_{F}\right) I\right)^{-1}=3.57$, indicating the strong SF exists in $\mathrm{MgCNi}_{3}$. Our results are very close to the previous theoretical reports ${ }^{12-15}$, which proves the validity of present calculations.

We firstly studied the A-site doping effect by investigating the electronic structures of $\mathrm{NaCNi}_{3}, \mathrm{MgCNi}_{3}$, $\mathrm{AlCNi}_{3}$, and $\mathrm{SiCNi}_{3}$. Using a doubled supercell, we also calculated the electronic structure of $\mathrm{Mg}_{0.5} \mathrm{Al}_{0.5} \mathrm{CNi}_{3}$. As shown in figure 3, the doping of A-site atoms does not change the overall shape of DOS of $\mathrm{ACNi}_{3}$ near $E_{F}$. The main change is the $E_{F}$ moving with the variation of the amount of electrons. Thus the doping effect of A- 
TABLE I. The $N\left(E_{F}\right)$ (states/eV/spin) in NM ground state (above "/") and Stoner parameter $I$ (under "/") of ACM 3 (A = $\mathrm{Na}, \mathrm{Mg}, \mathrm{Al}$, and $\mathrm{Si}, \mathrm{M}=3 d$ transition metal elements).

\begin{tabular}{|c|c|c|c|c|c|c|c|c|c|}
\hline $\begin{array}{ll}\mathbf{M} \rightarrow \\
\mathbf{A} \downarrow & \\
\end{array}$ & $\overline{\mathrm{Sc}}$ & $\overline{T i}$ & $\mathbf{V}$ & $\mathrm{Cr}$ & Mn & $\mathrm{Fe}$ & Co & $\mathrm{Ni}$ & $\overline{\mathrm{Cu}}$ \\
\hline $\mathrm{Na}$ & $3.66 / 0.26$ & $3.19 / 0.22$ & $1.51 / 0.50$ & $4.17 / 0.32$ & $7.84 / 0.37$ & $7.61 / 0.30$ & $3.83 / 0.41$ & $7.45 / 0.33$ & $0.44 / 0.74$ \\
\hline $\mathrm{Mg}$ & $5.30 / 0.30$ & $1.64 / 0.40$ & $4.28 / 0.23$ & $2.86 / 0.34$ & $5.47 / 0.35$ & $6.20 / 0.32$ & $3.69 / 0.41$ & $2.57 / 0.28$ & $0.41 / 0.79$ \\
\hline Al & $0.26 / 0.29$ & $1.491 / 0.05$ & $4.31 / 0.32$ & $2.22 / 0.32$ & $4.87 / 0.45$ & $5.53 / 0.39$ & $0.77 / 0.74$ & $1.13 / 0.58$ & $1.04 / 0.20$ \\
\hline $\mathrm{Si}$ & $1.26 / 0.42$ & $2.23 / 0.25$ & $6.38 / 0.21$ & $3.06 / 0.27$ & $6.20 / 0.47$ & $1.35 / 0.31$ & $3.87 / 0.42$ & $1.68 / 0.19$ & $0.75 / 0.72$ \\
\hline
\end{tabular}

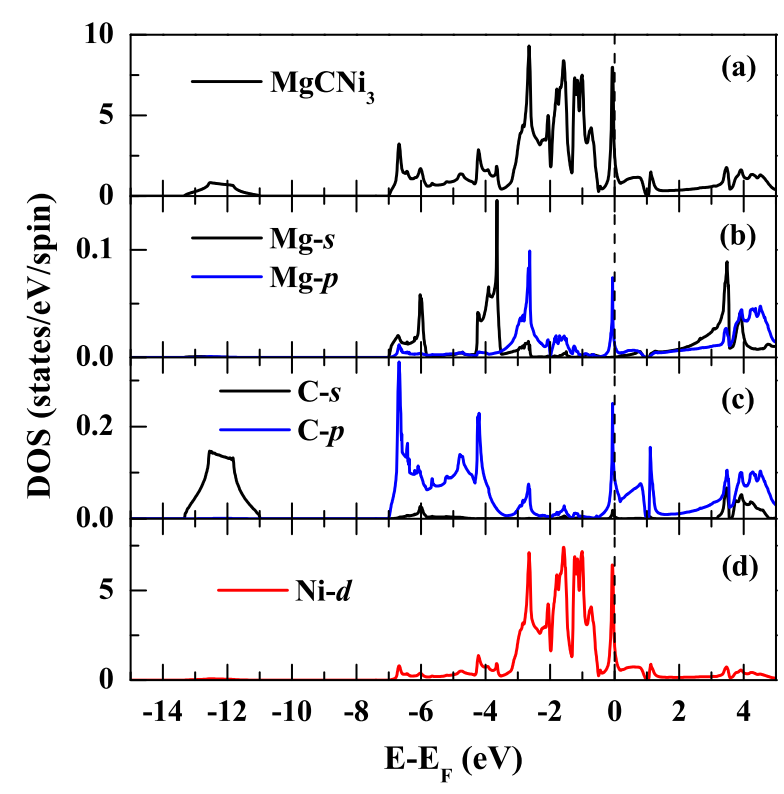

FIG. 2. Total and atom-orbital-projected DOS of $\mathrm{MgCNi}_{3}$.

site atom of $\mathrm{AXM}_{3}$ can be evaluated by the rigid band approximation. For $\mathrm{NaCNi}_{3}$, it can be seen as doped $\mathrm{MgCNi}_{3}$ by a hole, which causes $E_{F}$ decreasing. The $E_{F}$ just locates at the DOS peak, leading to very large $N\left(E_{F}\right)$, which makes $\mathrm{NaCNi}_{3}$ satisfied with the Stoner criteria $N\left(E_{F}\right) I>1$. Therefore, spin polarization appears in $\mathrm{NaCNi}_{3}$.

Next we consider the X-site doping effect. Figure 4 shows the DOS of $\mathrm{MgBNi}_{3}, \mathrm{MgCNi}_{3}$ and $\mathrm{MgNNi}_{3}$. Since the $\mathrm{X}-2 p$ electrons strongly hybridize with $\mathrm{Ni}-3 d$ electrons, the change of X-site atoms is expected to strongly influence the shape of DOS. The evaluation of X-site doping effect needs to investigate the X-M hybridization besides the $E_{F}$ moving. For instance, replacing the carbon atom in $\mathrm{MgCNi}_{3}$ by boron atom, $E_{F}$ moves to the DOS peak. On the other hand, the B-Ni hybridization makes the peak smeared, which does not lead to very large $N\left(E_{F}\right)$. Therefore $\mathrm{MgBNi}_{3}$ is still in the NM ground state. The calculated $N\left(E_{F}\right)$ of $\mathrm{MgBNi}_{3}$ is 2.58 states $/ \mathrm{eV} / \mathrm{spin}$, which can be comparable to that of $\mathrm{MgCNi}_{3}$. For $\mathrm{MgNNi}_{3}$, the DOS peak is smeared, too. Meanwhile $E_{F}$ moves away from the DOS peak, leading to small $N\left(E_{F}\right)=1.36$ states $/ \mathrm{eV} / \mathrm{spin}$.

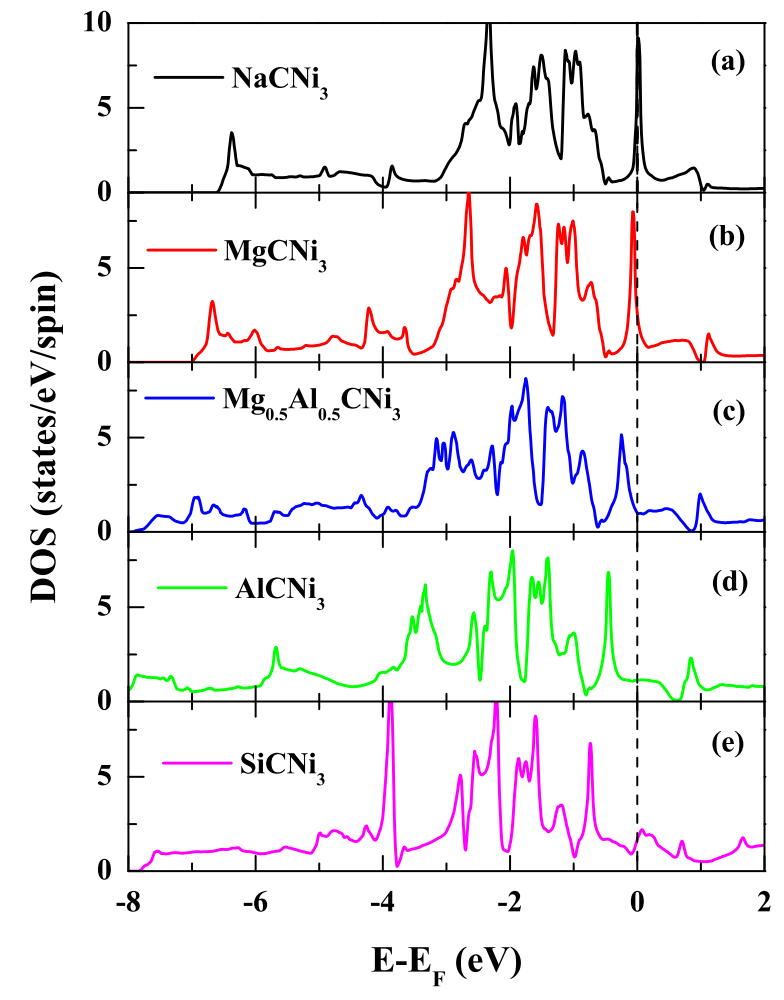

FIG. 3. DOS of (a) $\mathrm{NaCNi}_{3}$, (b) $\mathrm{MgCNi}_{3}$, (c) $\mathrm{Mg}_{0.5} \mathrm{Al}_{0.5} \mathrm{CNi}_{3}$, (d) $\mathrm{AlCNi}_{3}$, and (e) $\mathrm{SiCNi}_{3}$.

According to our calculation, except for $\mathrm{Ni}$ - and $\mathrm{Cu}-$ based $\mathrm{AXM}_{3}$, all the other compounds have similar character that the bonding and anti-bonding states are far from $E_{F}$. That means for most $\mathrm{AXM}_{3}$ compounds, there exists weak X-M hybridizations at $E_{F}$. Figure 5 shows the DOS of $\mathrm{AlCCr}_{3}$ and $\mathrm{AlNCr}_{3}$. The shapes of DOS near $E_{F}$ are very similar. The only difference is the location of $E_{F}$ decided by total electrons. Thus for most $\mathrm{AXM}_{3}$ (except for $\mathrm{Ni}$ - and $\mathrm{Cu}$-based ones) the influence of electronic structure by changing X-site atom can be approximately evaluated as hole or electron doping.

Now we study the M-site doping effect on the electron structure. Figure 6 shows the DOS of $\mathrm{MgCM}_{3}$. The shape of the DOS of $\mathrm{MgCZn}_{3}$ is quite different from those of other $\mathrm{MgCM}_{3}$. It is possibly due to that all the orbitals of $\mathrm{Zn}$ are fulfilled, which makes electrons of $\mathrm{Zn}$ atom localized. From $\mathrm{MgCSc}_{3}$ to $\mathrm{MgCCu}_{3}, E_{F}$ moves towards 


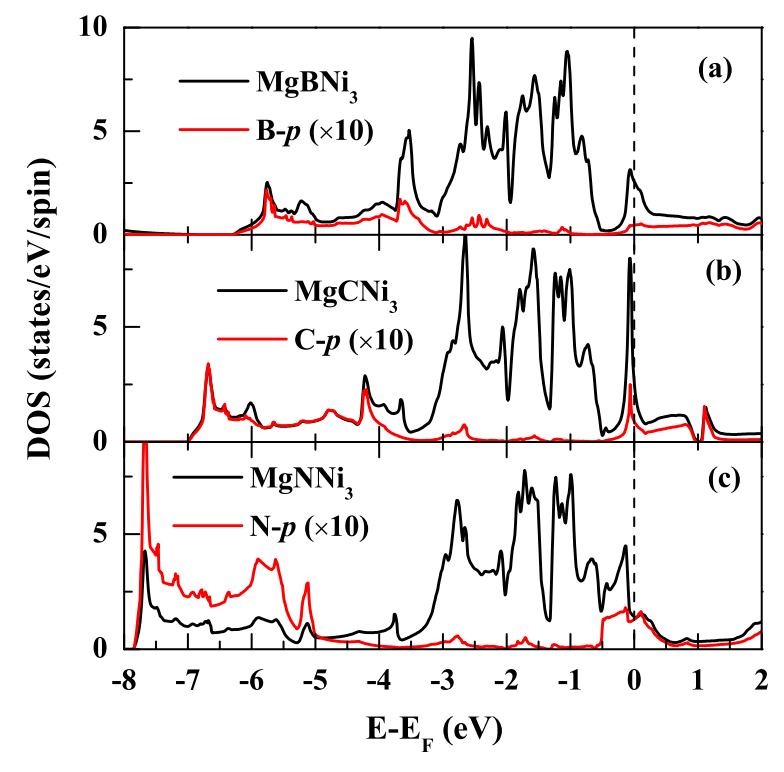

FIG. 4. DOS of (a) $\mathrm{MgBNi}_{3}$, (b) $\mathrm{MgCNi}_{3}$, and (c) $\mathrm{MgNNi}_{3}$.

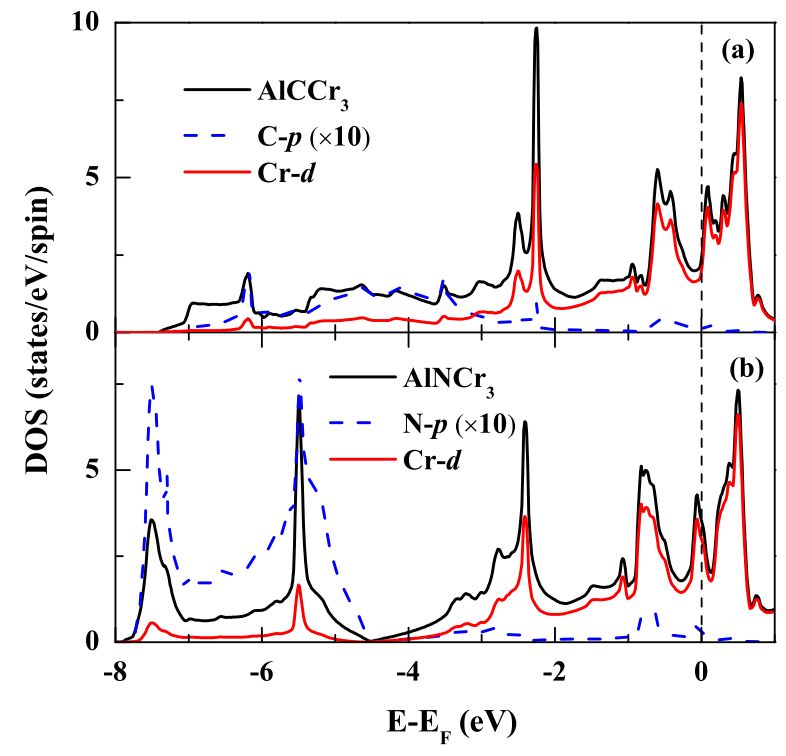

FIG. 5. DOS of (a) $\mathrm{AlCCr}_{3}$ and (b) $\mathrm{AlNCr}_{3}$.

to high energy as total electrons increase, and the shapes of DOS keep similar feature. Can we just deduce the Msite doping effect using rigid band approximation? The answer is negative.

We further investigated the electronic structure of doped $\mathrm{MgCNi}_{3}$ by $\mathrm{Co}$ (i.e. $\left.\mathrm{MgC}(\mathrm{Ni}, \mathrm{Co})_{3}\right)$. The calculated DOS in NM state of the doped $\mathrm{MgCNi}_{3}$ by one, two, and three cobalt atoms are shown in figures 7 (a)(d). $E_{F}$ of $\mathrm{MgCCo}_{3}$ locates at a DOS peak, which makes $\mathrm{MgCCo}_{3}$ be in FM ground state. For $\mathrm{MgCCoNi}_{2}$ and $\mathrm{MgCCo}_{2} \mathrm{Ni}$, the DOS near $E_{F}$ can be seen as the sum of the DOS of Ni-3d electrons and Co- $3 d$ electrons. The small $N\left(E_{F}\right)$ of $\mathrm{MgCCoNi}_{2}$ and $\mathrm{MgCCo}_{2} \mathrm{Ni}$ lead them

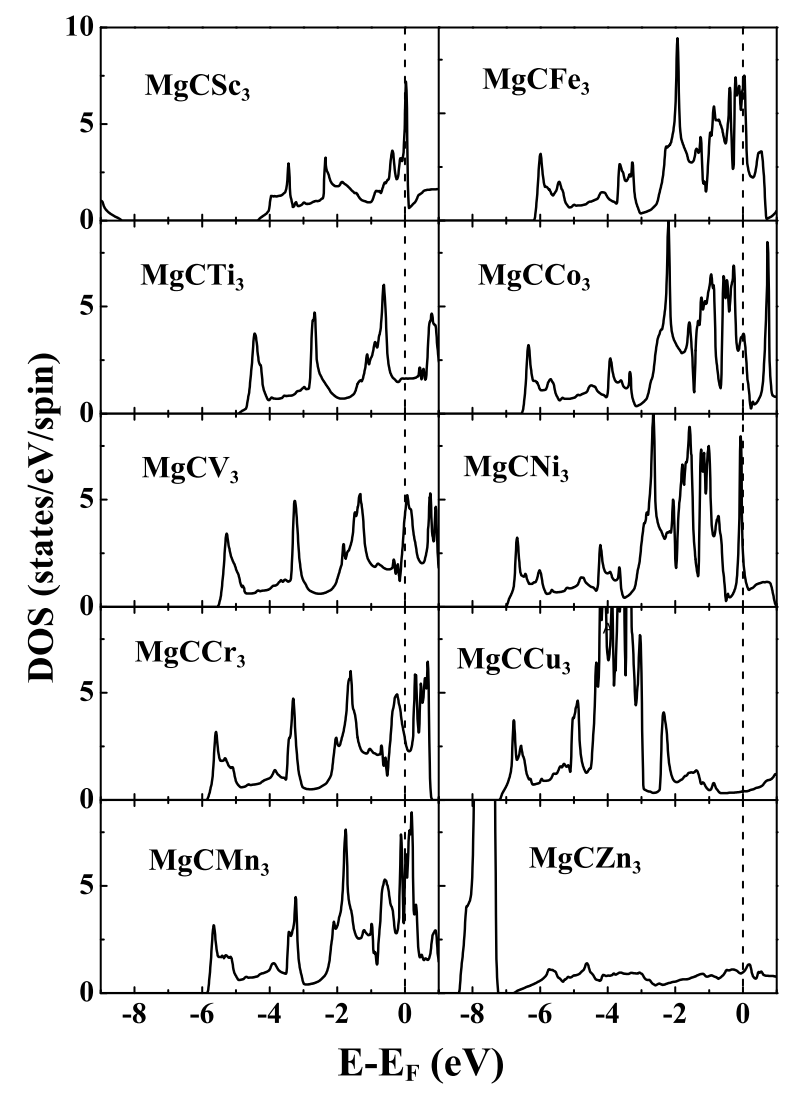

FIG. 6. DOS of $\mathrm{MgCM}_{3}(\mathrm{M}=3 d$ transition metal elements).

to show NM ground state. The DOS peak (van Hove singularity) splits into two peaks contributed by $\pi^{*}$ antibonding states of $\mathrm{C}-\mathrm{Ni}$ and $\mathrm{C}-\mathrm{Co}$, respectively. The Co doping decreases the total electrons, which reduces $E_{F}$ and makes the $\pi^{*}$ anti-bonding state of $\mathrm{C}-\mathrm{Ni}$ unoccupied. On the other hand, the Co doping weakens the DOS peak of Ni-3d electrons and enhances the DOS peak of Co-3d electrons. From the above discussion, one can deduce that as $\mathrm{MgCNi}_{3}$ is doped by a small Co content, the DOS peak of Ni-3d electrons can still has considerable strength. Meanwhile $E_{F}$ moves through the peak, which will lead to very large $N\left(E_{F}\right)$. According to Stoner criteria $N\left(E_{F}\right) I>1$, the system will become FM state. The calculation of $\mathrm{MgCCo}_{0.5} \mathrm{Ni}_{2.5}$ proves the deduction (figure 7 (e)). However such a FM state has not been observed in experiments $\frac{15}{}$, might due to a high doping content or an inaccurate stoichiometry. Similarly, the variations of $N\left(E_{F}\right)$ and magnetic properties of M-site doping in other $\mathrm{AXM}_{3}$ can be evaluated, too.

Additionally, the doping effect of some isovalent A-site atoms was also investigated. As shown in figure 8 , changing $\mathrm{Mg}$ to the isovalent atoms $\mathrm{Zn}$ and $\mathrm{Cd}$, the electronic structure near $E_{F}$ rarely changes. It supports the A-site atoms only give the effective valence electrons as Rosner et al. proposed $\frac{12}{2}$. Thus the doping effects of A-, X-, and M-sites we concluded above can be generally applied to most $3 d$ transition-metal based antiperovskites. 

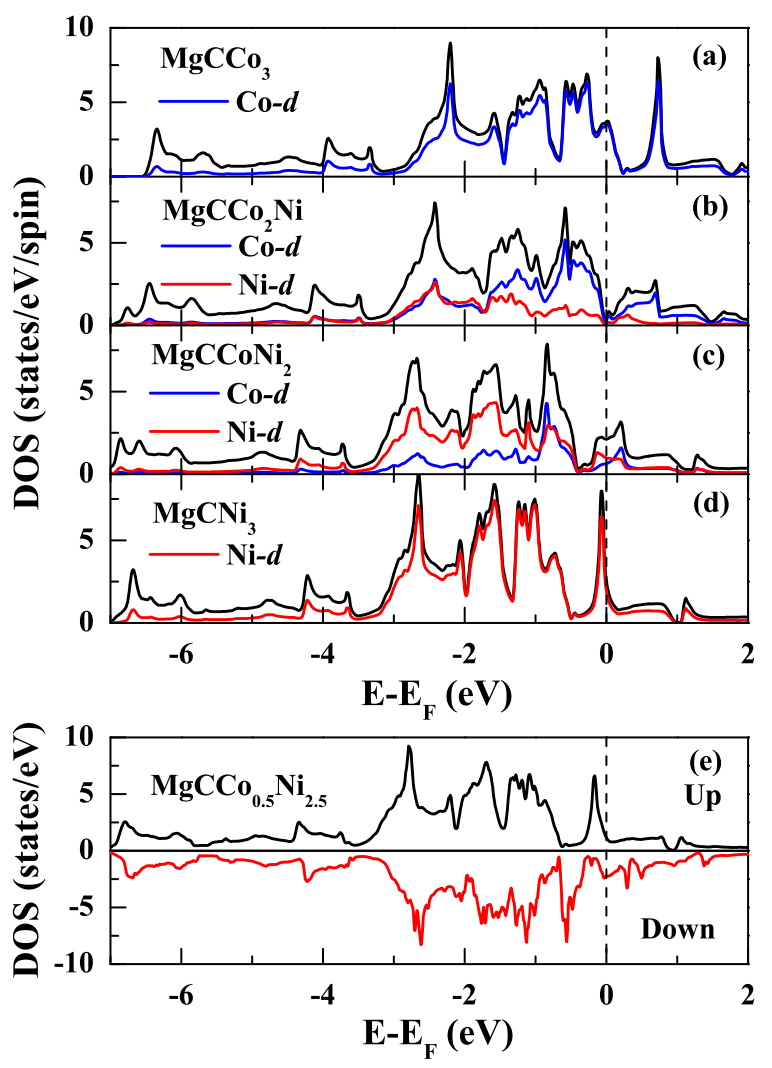

FIG. 7. DOS in NM state of (a) $\mathrm{MgCCo}_{3}$, (b) $\mathrm{MgCCo}_{2} \mathrm{Ni}$, (c) $\mathrm{MgCCoNi}_{2}$, (d) $\mathrm{MgCNi}_{3}$ and (e) DOS in FM state of $\mathrm{MgCCo}_{0.5} \mathrm{Ni}_{2.5}$.

\section{B. Magnetic phase diagram of $\mathrm{ACM}_{3}$}

We calculated the Stoner parameters $I$ of a series of carbon-based $\mathrm{ACM}_{3}$ (see table I). According to the doping effects we concluded above, the variation of $N\left(E_{F}\right)$ of different $\mathrm{AXM}_{3}$ can be evaluated. Using the factor $N\left(E_{F}\right) I$, we drew the magnetic phase diagram of carbonbased $\mathrm{ACM}_{3}$ in figure 9 .

The $\mathrm{ACM}_{3}$ locating in the areas with the colors green to red are satisfied with the Stoner criteria $N\left(E_{F}\right) I>1$ and therefore show FM ground state. All the Mn-based antiperovskites and most Fe-based antiperovskites show FM. Here we must point out that we only consider the NM and FM states. In reality, abundant magnetisms, e.g. antiferromagnetism and non-collinear magnetism, are observed experimentally in the Mn-based antiperovskites ${ }^{31}$. In this sense, our phase diagram can not present the real magnetism of such compounds. But in principle, if an itinerant system has very high $N\left(E_{F}\right)$ in NM state, this system is unstable and prefers to be spin-polarized. Accordingly, the magnetic ordering must occur to lower $N\left(E_{F}\right)$ to stabilize the system. Therefore, if a compound locates in the FM area of our phase diagram, it means that the NM state does not favor the energy minimum and the magnetic ordering will emerge. Such phase dia-

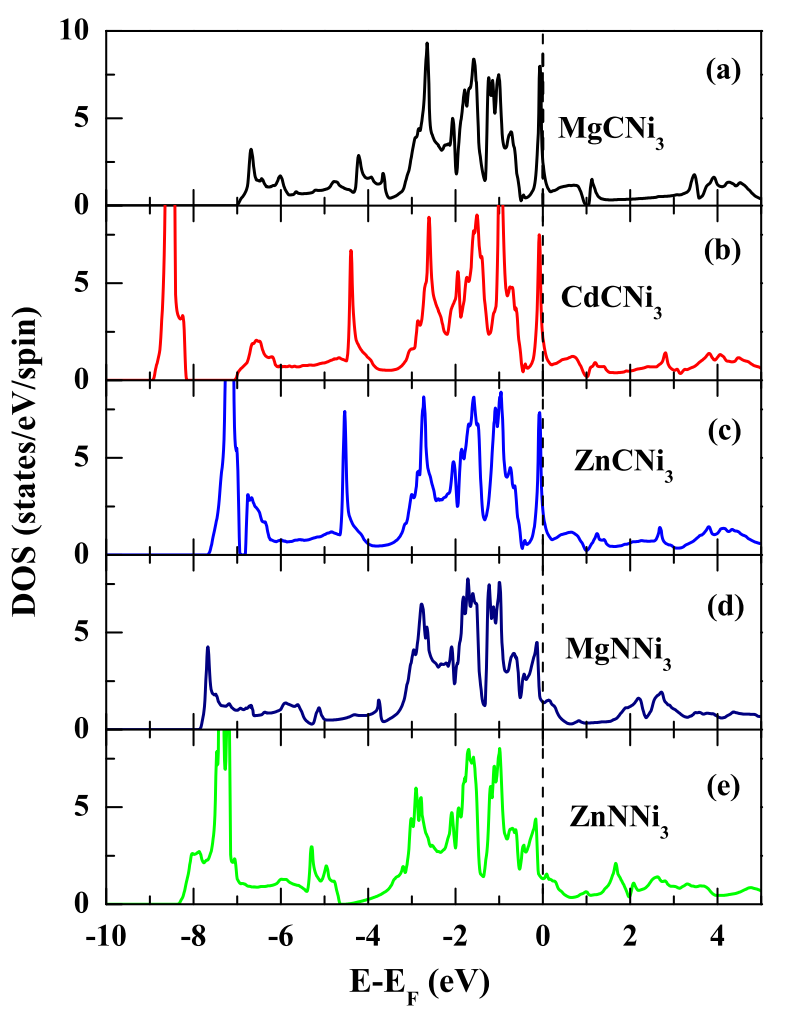

FIG. 8. DOS of (a) $\mathrm{MgCNi}_{3}$, (b) $\mathrm{CdCNi}_{3}$, (c) $\mathrm{ZnCNi}_{3}$, (d) $\mathrm{MgNNi}_{3}$, and (e) $\mathrm{ZnNNi}_{3}$.

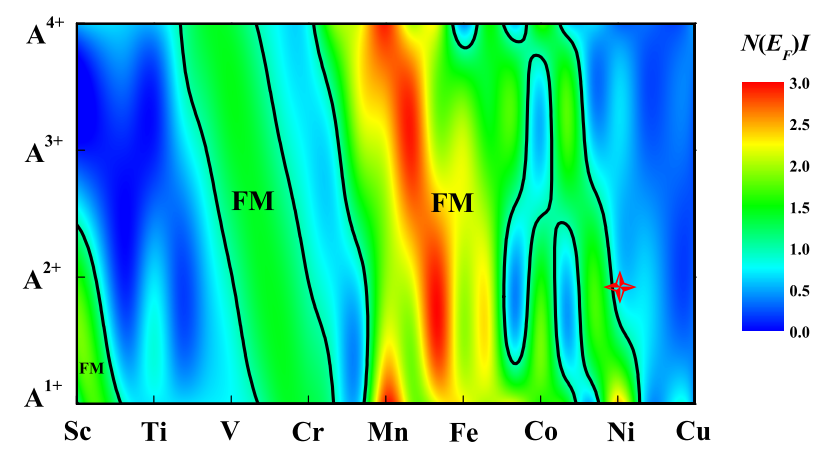

FIG. 9. Magnetic phase diagram of $\mathrm{ACM}_{3}$. The black line is the NM-FM boundary. $\mathrm{MgCNi}_{3}$ is highlighted using the red star.

gram can help to explore new magnetic antiperovskites. Furthermore one can also predict the magnetic state of most boron- and nitrogen-based antiperovskites based on the magnetic phase diagram after considering the $\mathrm{X}$-site doping effect as concluded above.

We highlighted $\mathrm{MgCNi}_{3}$ using a red star in the phase diagram. Obviously, $\mathrm{MgCNi}_{3}$ is very close to the $\mathrm{FM}$ state. One can imagine that there must be some interplay between $\mathrm{FM}$ and $\mathrm{SC}$ in $\mathrm{MgCNi}_{3}$. As mentioned above, in order to figure out such interplay, exploring more $\mathrm{MgCNi}_{3}$-like superconducting antiperovskites is necessary. We assume SC may appear in the area near the 
NM-FM boundary, since in such area the compounds have sizeable $N\left(E_{F}\right)$ that can lead to a strong EPC.

\section{Superconductivity in $\mathrm{MgCNi}_{3}$ and $\mathrm{AXCr}_{3}$}

In the magnetic phase diagram, one can notice that some Cr-based antiperovskites locate in a small interval between two FM areas, which makes $\mathrm{AXCr}_{3}$ as a good system to prove our deduction above. In this part, we investigated the potential $\mathrm{SC}$ in $\mathrm{AXCr}_{3}$. For comparison, the lattice dynamics and $\mathrm{EPC}$ property of $\mathrm{MgCNi}_{3}$ were also calculated.

Previously, we calculated the formation energies and electronic structures of a series of $\mathrm{Cr}$-based antiperovskite carbides $\mathrm{ACCr}_{3}{ }^{47}$. Only $\mathrm{AlCCr}_{3}$ and $\mathrm{GaCCr}_{3}$ have negative formation energies and may be synthesized experimentally. Both the two compounds show the NM ground state. Because of the isovalent A-site atoms, the electronic structures near $E_{F}$ of the two compounds are almost the same. Similar to $\mathrm{MgCNi}_{3}, E_{F}$ of the two compounds locates at a slope of a DOS peak, which leads to large $N\left(E_{F}\right)$ and may generate sizeable EPC.

In present work, we also investigated a series of $\mathrm{Cr}-$ based antiperovskite nitrides $\mathrm{ANCr}_{3}$. The lattice parameters and the formation energies of NM and FM states are listed in table II. It can be seen that the magnetic state of $\mathrm{A}^{n+} \mathrm{NCr}_{3}$ is almost the same as that of $\mathrm{A}^{(n+1)+} \mathrm{CCr}_{3}$, which accords with the X-site doping effect we concluded above. The calculated results show that $\mathrm{ZnNCr}_{3}, \mathrm{AlNCr}_{3}, \mathrm{GaNCr}_{3}$, and $\mathrm{SnNCr}_{3}$ have negative formation energies and therefore may be synthesized under normal condition in experiments.

The overall shapes of DOS near $E_{F}$ of $\mathrm{ZnNCr}_{3}$, $\mathrm{AlNCr}_{3}, \mathrm{GaNCr}_{3}$, and $\mathrm{SnNCr}_{3}$ are very similar (see figures 10 (a)-(d)). The calculated $N\left(E_{F}\right)$ and Stoner criteria $N\left(E_{F}\right) I$ are listed in table III] It was previously suggested that $\mathrm{GaNCr}_{3}$ may be a potential superconductor ${ }^{34,36}$. According to our calculations, it can be seen that $N\left(E_{F}\right) I \approx 1$ for $\mathrm{AlNCr}_{3}$ and $\mathrm{GaNCr}_{3}$, which means the two compounds just locate at the FM quantum critical point. They show weak FM with small exchange splitting energies (figures 10 (e) and (f)). Therefore we did not consider the possibility of SC in $\mathrm{AlNCr}_{3}$ and $\mathrm{GaNCr}_{3}$. It is worth noting that $E_{F}$ of $\mathrm{ZnNCr}_{3}$ locates very close to a DOS peak, which leads to large $N\left(E_{F}\right)$ and suggests a sizeable EPC.

We calculated the lattice dynamics and EPC properties of $\mathrm{MgCNi}_{3}, \mathrm{AlCCr}_{3}, \mathrm{GaCCr}_{3}$, and $\mathrm{ZnNCr}_{3}$. For reliability, we tested different exchange-correlation potentials and calculation parameters. The results are coincide with each other. The calculated phonon dispersion curves and the corresponding atom-projected phonon DOS are shown in figure 11. The ideal antiperovskite in cubic structure $(\mathrm{Pm} \overline{3} \mathrm{~m})$ with five atoms per unit cell presents fifteen phonon modes including three acoustic and twelve optical modes. The highest three optical branches resulting mainly from the lighter $\mathrm{C} / \mathrm{N}$ atom vibrations are well
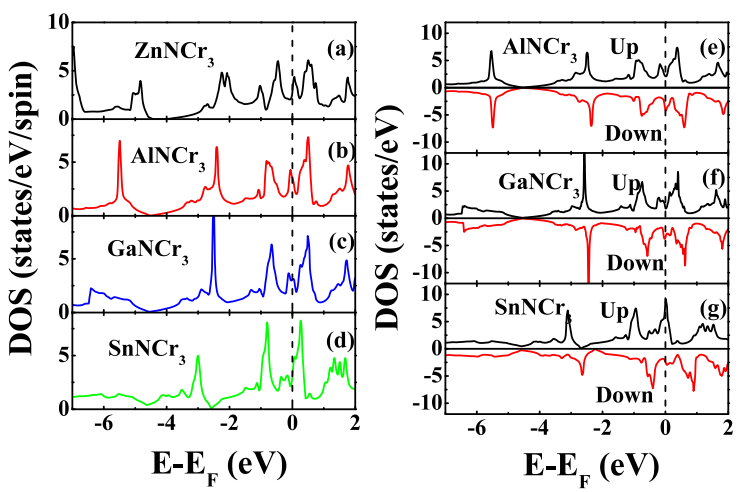

FIG. 10. Left panel: DOS of (a) $\mathrm{ZnNCr}_{3}$, (b) $\mathrm{AlNCr}_{3}$, (c) $\mathrm{GaNCr}_{3}$, and (d) $\mathrm{SnNCr}_{3}$ in NM state. Right panel: DOS of (e) $\mathrm{AlNCr}_{3}$, (f) $\mathrm{GaNCr}_{3}$, and (g) $\mathrm{SnNCr}_{3}$ in $\mathrm{FM}$ state.

separated from the other phonon branches. The phonon modes in low-frequency region come mainly from the vibrations of $\mathrm{Cr} / \mathrm{Ni}$ atoms with partial contribution of $\mathrm{A}$ and $\mathrm{C} / \mathrm{N}$ vibrations.

Earlier calculations suggested lattice instabilities existing in $\mathrm{MgCNi}_{3}{ }^{48}-51$, which seem to be ruled out by the recent experimental and theoretical reports 24,25 . According to our calculation, no imaginary frequency was found in $\mathrm{MgCNi}_{3}$, and the result is similar to the experimental one ${ }^{24}$. Soft longitudinal acoustic (LA) modes at $\mathrm{M}$ and $\mathrm{R}$ points are reproduced. Between $\mathrm{X}$ and $\mathrm{R}$ point, transverse acoustic (TA) mode of $\mathrm{MgCNi}_{3}$ is significantly softened and very close to instability. In

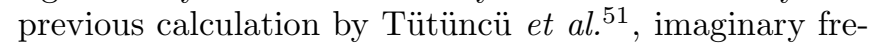
quency exists just in the same $q$-space path. Unfortunately, there are no existing experimental data about the phonon properties in such place. Additionally, in many previous calculations $48,49,51$, strong softening or imaginary frequency is shown between $\Gamma$ and $R$ points. Our calculation reproduces such softening. However, experimental result only shows very small hints of the softening of TA mode between $\Gamma$ and $\mathrm{R}$ points 24 . The differences between theoretical and experimental results may be due to the sample quality. As Heid et al $\stackrel{49}{\underline{4}}$ pointed out, the lattice defects may play an important role in stabilizing the real structure. Indeed, the stoichiometry of the reported single crystals are deficient $\left(\mathrm{MgCNi}_{2.8}\right.$ and $\mathrm{MgC}_{0.92} \mathrm{Ni}_{2.88}$ ), which may be the reason of the absence of such soft-phonon anomaly in the experimental result. Such phonon mode softening is considered as the key role in the $\mathrm{SC}$ mechanism of $\mathrm{MgCNi}_{3}$ 48,52,53. In experiments, single crystals with better quality are needed to figure out such puzzling anomalies.

For $\mathrm{AlCCr}_{3}, \mathrm{GaCCr}_{3}$, and $\mathrm{ZnNCr}_{3}$, the absence of imaginary frequency suggests the dynamic stability of the considered crystal structures of the three compounds. The acoustic modes are harder than those of $\mathrm{MgCNi}_{3}$, and no modes are close to instability. The optical phonons at $\Gamma$ point belong to the following irreducible representations: $F\left(O_{h}\right)=3 F_{1 u}+F_{2 u}$ (see figure 12), 
TABLE II. Lattice parameter $a$, formation energies $\triangle E$, and magnetic moments per Cr atom $M$ of $\mathrm{ANCr}_{3}$.

\begin{tabular}{ccccccccc}
\hline \hline $\mathrm{A}$ & $\mathrm{Zn}$ & $\mathrm{Ga}$ & $\mathrm{Al}$ & $\mathrm{Ag}$ & $\mathrm{Cd}$ & $\mathrm{Sn}$ & $\mathrm{Mg}$ & $\mathrm{In}$ \\
\hline$a(\AA)$ & 3.861 & 3.868 & 3.878 & 3.861 & 3.91 & 3.944 & 3.956 & 3.918 \\
$\triangle E_{N M}(\mathrm{eV} /$ atom $)$ & -0.1027 & -0.2195 & -0.2448 & 0.0972 & 0.0619 & -0.0813 & 0.0173 & 0.0187 \\
$\triangle E_{F M}(\mathrm{eV} /$ atom $)$ & -0.1027 & -0.2206 & -0.2455 & 0.0969 & 0.0626 & -0.0831 & 0.0173 & 0.0186 \\
$M\left(\mu_{B} / \mathrm{Cr}\right)$ & 0 & 0.23 & 0.22 & 0.06 & 0 & 0.69 & 0.03 & 0.4 \\
\hline \hline
\end{tabular}

TABLE III. Calculated $N\left(E_{F}\right)$ (states/eV/spin) and Stoner criteria $N\left(E_{F}\right) I$ of $\mathrm{ZnNCr}_{3}, \mathrm{AlNCr}_{3}, \mathrm{GaNCr}_{3}$, and $\mathrm{SnNCr}_{3}$.

\begin{tabular}{lcccc}
\hline \hline & $\mathrm{ZnNCr}_{3}$ & $\mathrm{AlNCr}_{3}$ & $\mathrm{GaNCr}_{3}$ & $\mathrm{SnNCr}_{3}$ \\
\hline$N\left(E_{F}\right)$ & 2.81 & 3.53 & 3.08 & 3.01 \\
$N\left(E_{F}\right) I$ & 0.76 & 1.09 & 1.01 & 1.29 \\
\hline
\end{tabular}

TABLE IV. Optical phonon frequencies $\omega(\mathrm{THz})$ at $\Gamma$ point, the calculated EPC strengths $\lambda$, and the logarithmically averaged phonon frequency $\omega_{l o g}(\mathrm{~K})$.

\begin{tabular}{|c|c|c|c|c|c|c|}
\hline & $\omega\left(F_{1 u}^{1}\right)$ & $\omega\left(F_{1 u}^{2}\right)$ & $\omega\left(F_{1 u}^{3}\right)$ & $\omega\left(F_{2 u}\right)$ & $\lambda$ & $\omega_{l o g}$ \\
\hline $\mathrm{MgCNi}_{3}$ & 4.26 & 5.21 & 17.01 & 8.28 & 1.34 & 115.77 \\
\hline $\mathrm{AlCCr}_{3}$ & 4.87 & 8.57 & 21.84 & 5.48 & 0.60 & 291.51 \\
\hline $\mathrm{GaCCr}_{3}$ & 4.19 & 5.62 & 21.63 & 4.44 & 0.78 & 253.91 \\
\hline $\mathrm{ZnNCr}_{3}$ & 2.45 & 5.92 & 18.98 & 4.82 & 0.67 & 260.27 \\
\hline
\end{tabular}

which are infrared (IR) active (table IV]). The $\mathrm{C} / \mathrm{N}$ related $F_{1 u}^{1}$ mode and $F_{1 u}^{3}$ mode of $\mathrm{ZnNCr}_{3}$ are softer than those of $\mathrm{AlCCr}_{3}$ and $\mathrm{GaCCr}_{3}$ maybe due to the larger atomic mass of nitrogen. We did not observe the significant softening of $F_{2 u}$ mode that reported by Tütüncü et al. in $\mathrm{RhNCr}_{3}$ and $\mathrm{GaNCr}_{3}{ }^{35,36}$.

The calculated Eliashberg functions $\alpha^{2} F(\omega)$ of $\mathrm{MgCNi}_{3}, \mathrm{AlCCr}_{3}, \mathrm{GaCCr}_{3}$, and $\mathrm{ZnNCr}_{3}$ are plotted in figure 13 according to the below formula:

$\alpha^{2} F(\omega)=\frac{1}{N(0)} \sum_{\mathbf{k}, \mathbf{q}, \nu, n, m} \delta\left(\epsilon_{\mathbf{k}}^{n}\right) \delta\left(\epsilon_{\mathbf{k}+\mathbf{q}}^{m}\right)\left|g_{\mathbf{k}, \mathbf{k}+\mathbf{q}}^{\nu, n}\right|^{2} \delta\left(\omega-\omega_{\mathbf{q}}^{\nu}\right)$,

where $\omega_{\mathbf{q}}^{\nu}$ are phonon frequencies, $\epsilon_{\mathbf{k}}^{n}$ electronic energies, and $g_{\mathbf{k}, \mathbf{k}+\mathbf{q}}^{\nu, n, m} E P$ matrix elements. The total EPC strength is

$$
\lambda=\sum_{\mathbf{q}, \nu} \lambda_{\mathbf{q}}^{\nu}=2 \int_{0}^{\infty} \frac{\alpha^{2} F(\omega)}{\omega} d \omega .
$$

The calculated $\lambda_{\mathbf{q}}^{\nu}$ are visualized as red circles in figure 11. For $\mathrm{MgCNi}_{3}$, the absence of imaginary frequency allows the direct calculation of the quantitative EPC contribution details from the lowest acoustic mode. Obviously for $\mathrm{MgCNi}_{3}$ the EPC originates mainly from acoustic phonon modes softening. For $\mathrm{AlCCr}_{3}, \mathrm{GaCCr}_{3}$, and $\mathrm{ZnNCr}_{3}$, optical modes are playing an important role. The EPC distributes throughout some low-frequency phonon modes. One can note that a larger $\lambda_{\mathbf{q}}^{\nu}$ always exists at the frequencies at which large Cr-phonon DOS

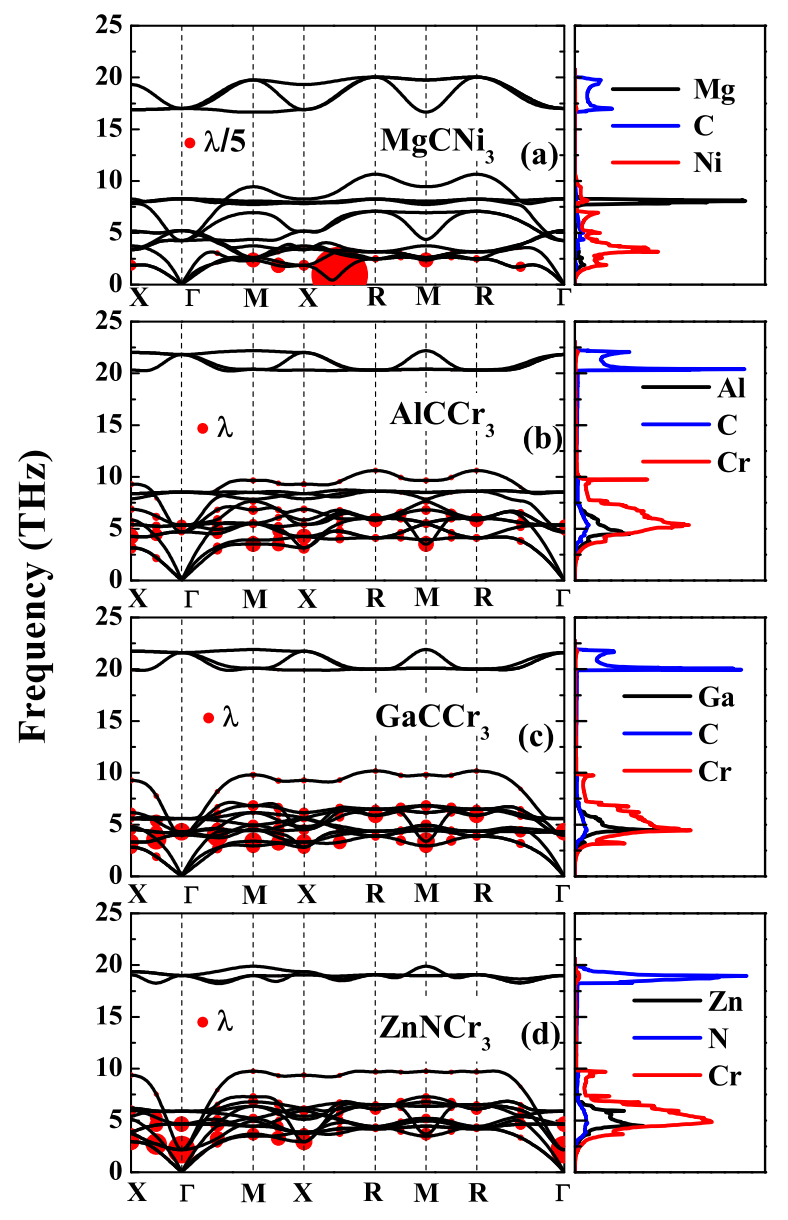

FIG. 11. Phonon dispersions and phonon DOS of (a) $\mathrm{MgCNi}_{3}$, (b) $\mathrm{AlCCr}_{3}$, (c) $\mathrm{GaCCr}_{3}$, (d) $\mathrm{ZnNCr}_{3}$. The phonon dispersions are decorated with symbols, proportional to the partial EPC strength $\lambda_{q v}$.

present. At the zone center, the $F_{1 u}^{1}$ and $F_{2 u}$ modes mainly contribute to EPC. For the two modes, Cr atoms vibrate strongly against each other (figure 12). Such vibrations can make large change in the overlap of $3 d$ orbitals between neighbouring $\mathrm{Cr}$ atoms, leading to strong coupling. Comparing the EPC strength from different phonon modes of $\mathrm{GaCCr}_{3}$ and $\mathrm{ZnNCr}_{3}$, it can be found that the softening of $F_{1 u}^{1}$ mode can strongly enhance $\lambda$.

The integrated EPC strengths $\lambda(\omega)$ are calculated using equation (2), and are plotted in figure 13. The EPC comes mainly from the $\alpha^{2} F(\omega)$ peaks at low frequen- 


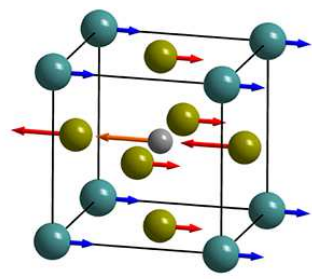

$F_{1 u}^{1}$

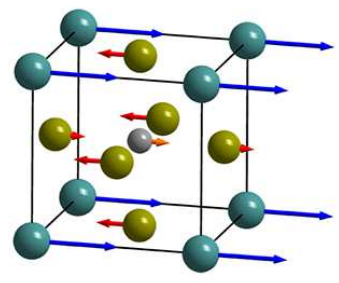

$F_{1 u}^{2}$

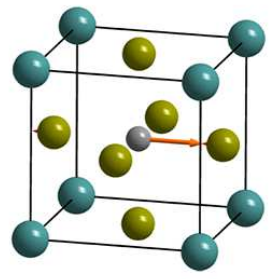

$F_{1 u}^{3}$

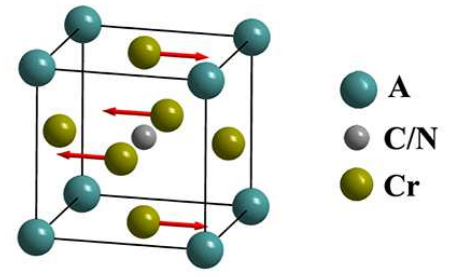

$F_{2 u}$

FIG. 12. Schematic eigen displacements of zone-centre optical phonon modes in $\mathrm{AXM}_{3}$. All phonon modes are infrared active.

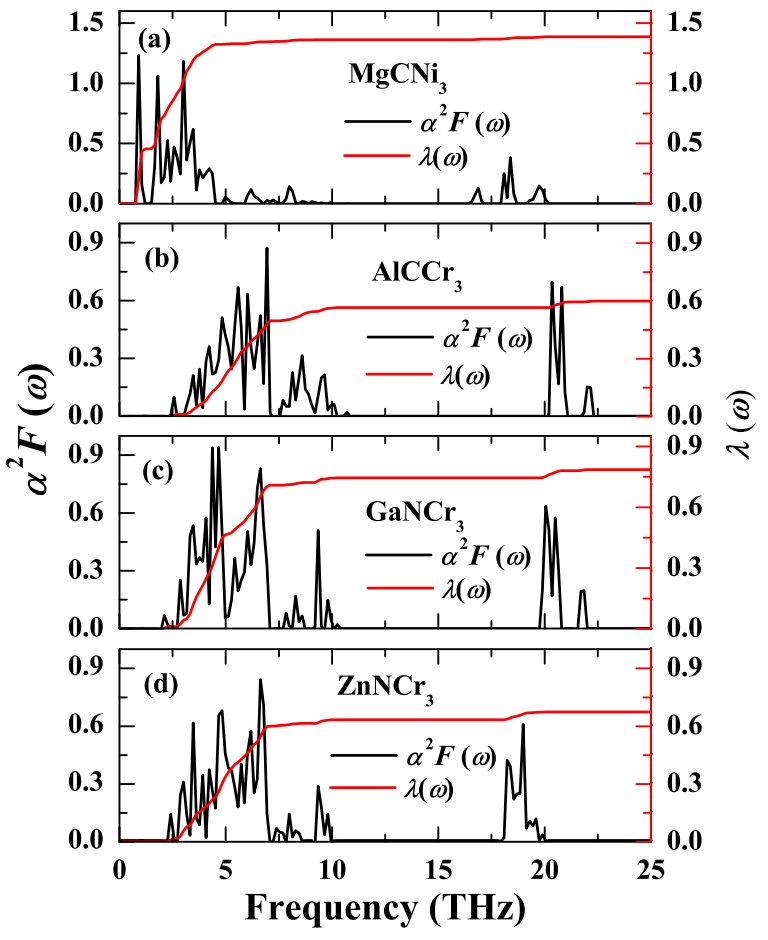

FIG. 13. Eliashberg functions (left) and frequency-dependent EPC strength $\lambda$ (right) of (a) $\mathrm{MgCNi}_{3}$, (b) $\mathrm{AlCCr}_{3}$, (c) $\mathrm{GaCCr}_{3}$, and (d) $\mathrm{ZnNCr}_{3}$.

cies, which are mostly related to the vibrations of $\mathrm{Ni} / \mathrm{Cr}$ atoms. For the peaks at high frequencies related to the vibrations of $\mathrm{C} / \mathrm{N}$ atoms, their contributions to $\lambda$ are less than $6 \%$.

The calculated $\lambda$ of $\mathrm{MgCNi}_{3}$ is 1.34 , implying very strong EPC. However, the calculated $\lambda$ of $\mathrm{AlCCr}_{3}$, $\mathrm{GaCCr}_{3}$, and $\mathrm{ZnNCr}_{3}$ are less than 1.0 and about $0.6 \sim$ 0.8 (table IV), implying moderate EPC. The difference of $\lambda$ for $\mathrm{AXCr}_{3}$ can be described qualitatively using Hopfield expression

$$
\lambda=\frac{N\left(E_{F}\right) I^{2}}{M\left\langle\omega^{2}\right\rangle}
$$

where $I$ is mean square EPC matrix element averaged over Fermi surface, $M$ is the ion mass, and $\left\langle\omega^{2}\right\rangle$ is the
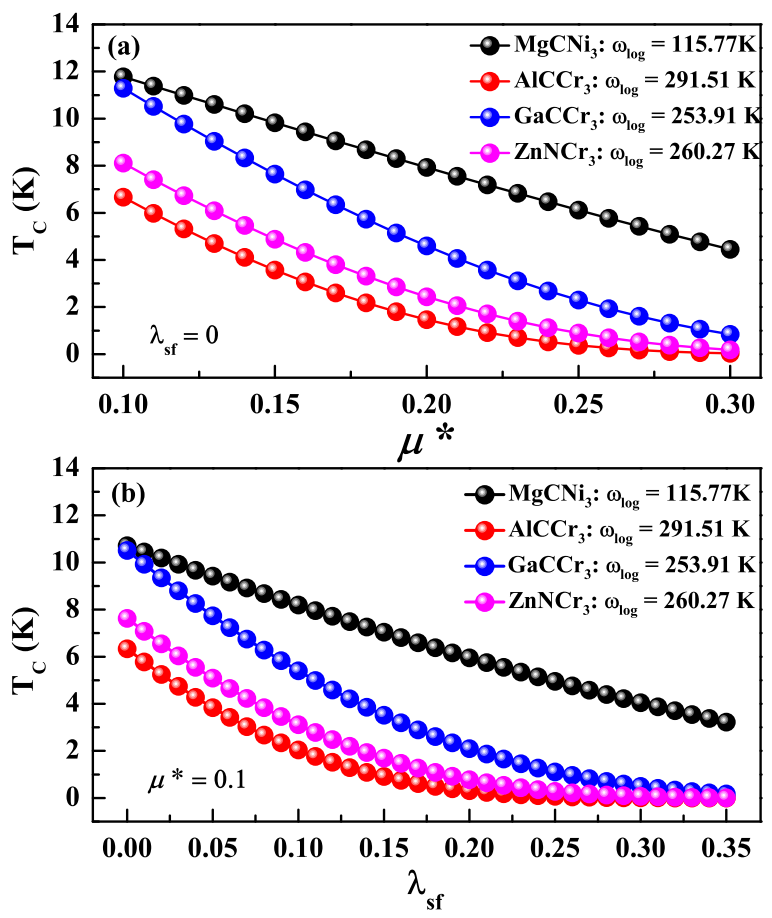

FIG. 14. (a) Variation of $T_{C}$ with $\mu^{*}$ (purely static pair breaking); (b) Variation of $T_{C}$ with $\lambda_{s f}$ (electron-paramagnon coupling).

average squared phonon frequency. The higher $N\left(E_{F}\right)$ and the lower phonon frequency make $\mathrm{GaCCr}_{3}$ has the larger $\lambda$.

To calculate $T_{C}$, we used the Allen-Dynes equation ${ }^{54-56}$

$$
T_{c}=\frac{\omega_{\log }}{1.20} \exp \left(-\frac{1.04(1+\lambda)}{\lambda-\mu^{*}-0.62 \lambda \mu^{*}}\right),
$$

where $\mu^{*}$ is Coulomb pseudopotential and the logarithmically averaged characteristic phonon frequency $\omega_{l o g}$ is defined as

$$
\omega_{l o g}=\exp \left(\frac{2}{\lambda} \int \frac{d \omega}{\omega} \alpha^{2} F(\omega) \log \omega\right) .
$$

At the absence of SF, using a typical $\mu^{*}=0.1$, we can get $T_{C}$ with the values of $11.82,6.67,11.29$, and $8.23 \mathrm{~K}$ for 
$\mathrm{MgCNi}_{3}, \mathrm{AlCCr}_{3}, \mathrm{GaCCr}_{3}$, and $\mathrm{ZnNCr}_{3}$, respectively. According to the experimental results of $\mathrm{MgCNi}_{3}$ single crystals $16.17 .20-24$, the observed $T_{C}$ is around $7 \mathrm{~K}$. It seems that our calculation overestimates $T_{C}$ of $\mathrm{MgCNi}_{3}$. Therefore we need to consider the influence of SF in the system.

We firstly considered the purely static pair breaking due to SF by varying $\mu^{*}$. In figure 14 (a), one can note that the increasing $\mu^{*}$ significantly depresses $T_{C}$. As $T_{C}$ around $7 \mathrm{~K}$, we can get $\mu^{*} \approx 0.23$. Such a value is smaller than the previous derivations of $\mu^{*}=0.33^{\underline{48}}$ and $\mu^{*}=$ $0.41^{52}$, but still larger than the typical values $\mu^{*}=0.1 \sim$ 0.15 for the absence of SF.

It is better to discuss the influence of SF in terms of electron-paramagnon coupling strength $\lambda_{s f} \underline{48}$. In that case, the effective coupling strength should be $\lambda_{\Delta}=$ $\lambda-\lambda_{s f}$. The effective mass of the carriers should be enhanced by the factor $1+\lambda_{Z}=1+\lambda+\lambda_{s f}$. And equation (4) shound be changed as 53,57

$$
T_{C}=\frac{\omega_{\log }}{1.45} \exp \left(-\frac{\left(1+\lambda_{Z}\right)}{\lambda_{\Delta}-\mu^{*}\left(1+0.5 \frac{\lambda_{\Delta}}{1+\lambda_{Z}}\right)}\right) .
$$

Figure 14 (b) shows the calculated $T_{C}$ using equation (6) with different $\lambda_{s f}$ (here the Coulomb pseudopotential is fixed to the typical value $\mu^{*}=0.1$ ). When $T_{C}$ is around $7 \mathrm{~K}$, we can get $\lambda_{s f} \approx 0.15$. Although the obtained parameter is small, it still implies that SC in the system is partially suppressed by depairing effect from $\mathrm{SF}^{52,53}$. The influence of $\mathrm{SF}$ on $T_{C}$ of $\mathrm{AlCCr}_{3}, \mathrm{GaCCr}_{3}$, and $\mathrm{ZnNCr}_{3}$ is also present in figure 14. If we consider the same SF influence as in $\mathrm{MgCNi}_{3}$, using equation (4) with $\mu^{*}=0.23$ and $\lambda_{s f}=0$, we can get $T_{C}=0.70,3.11$, and $1.40 \mathrm{~K}$ for $\mathrm{AlCCr}_{3}, \mathrm{GaCCr}_{3}$, and $\mathrm{ZnNCr}_{3}$, respectively; using equation (6) with $\mu^{*}=0.1$ and $\lambda_{s f}=0.15$, we can get $T_{C}=0.91,3.52$, and $1.69 \mathrm{~K}$ for $\mathrm{AlCCr}_{3}, \mathrm{GaCCr}_{3}$, and $\mathrm{ZnNCr}_{3}$, respectively.

Let us look back at the electronic structures of $\mathrm{AlCCr}_{3}$, $\mathrm{GaCCr}_{3}$, and $\mathrm{ZnNCr}_{3}$. The $E_{F}$ locates between two DOS peaks, which suggests in the system both hole and electron doping can enhance the SF and lead to a transition from SC to magnetic instability. Considering it has not been successful so far to investigate such transition in doped antiperovskites such as $\mathrm{Mg}_{1-x}\left(\mathrm{~A}^{1+}\right)_{x} \mathrm{CNi}_{3}$ due to the difficulty in experimental preparation of samples, $\mathrm{AXCr}_{3}$ may be good candidates to figure out the relation between SC and magnetism in antiperovskites.

\section{Other potential superconducting $\mathrm{AXM}_{3}$}

Since the discovery of $\mathrm{SC}$ in $\mathrm{MgCNi}_{3}$, researchers have made great efforts to explore other $3 d$ transitionmetal based antipervoskite superconductor ${ }^{32-36,58}$. The observed superconducting trace at $4.5 \mathrm{~K}$ found in $\mathrm{In}_{1.3} \mathrm{~B}_{0.7} \mathrm{Sc}_{3}$ is encouraging 32 . Theoretically, $\mathrm{InBSc}_{3}$ is close to weak FM and with an EPC superconducting

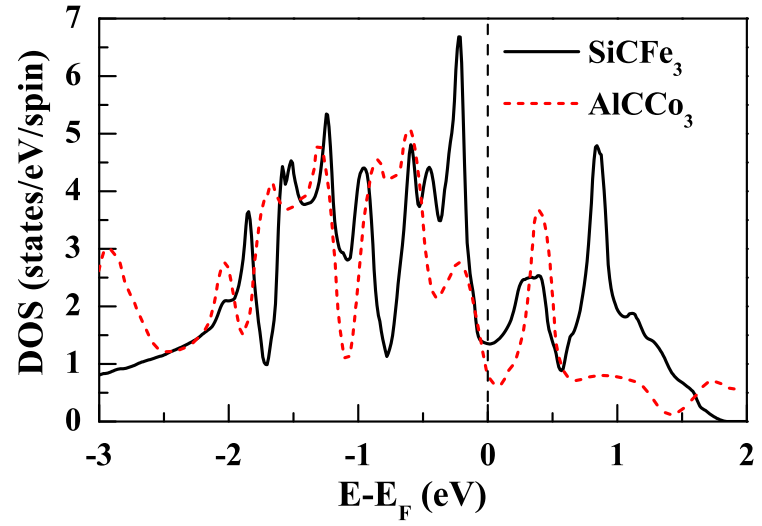

FIG. 15. DOS near $E_{F}$ of (a) $\mathrm{SiCFe}_{3}$ and (b) $\mathrm{AlCCo}_{3}$.

mechanism ${ }^{32.33}$. One should believe the Ni-based antiperovskite superconductor are not alone and other superconducting antiperovskites may be hidden behind them.

Indeed, our magnetic phase diagram shows that some $\mathrm{AXSc}_{3}$ should be very close to $\mathrm{FM}$, which may potentially be superconducting. There are also some other antiperovskite compounds close to $\mathrm{FM}$, such as $\mathrm{AXV}_{3}$, for which the cubic phase has not been successfully prepared so far. Here we present two potential superconducting parent materials $\mathrm{A}^{4+} \mathrm{CFe}_{3}(\mathrm{~A}=\mathrm{Si}, \mathrm{Ge}$, etc. $)$ and $\mathrm{A}^{3+} \mathrm{CCo}_{3}(\mathrm{~A}$ $=\mathrm{Al}, \mathrm{Ga}$, etc.). The DOS near $E_{F}$ of $\mathrm{SiCFe}_{3}$ and $\mathrm{AlCCo}_{3}$ are plotted in figure 15. One can notice that the $E_{F}$ of the two compounds just locates at a DOS valley. Thus both electron and hole doping can move $E_{F}$ to the DOS peaks nearby, and largely enhance $N\left(E_{F}\right)$ to yield strong EPC. Therefore, doped $\mathrm{A}^{3+} \mathrm{CCo}_{3}$ may be new Co-based

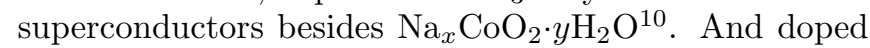
$\mathrm{A}^{4+} \mathrm{CFe}_{3}$ may be new Fe-based superconductors.

As we know, the role of EPC in the Fe-based superconductors is still puzzling. For instance, EPC mechanism can get a reasonable $T_{C}$ of the hcp iron under pressure, but can not explain the rapid disappearance of SC above $30 \mathrm{GPa}^{7,59,60}$. For the iron pnictide superconductors ${ }^{8}$, Boeri et al $\underline{\underline{61}, 62}$ and Mazin et al $\stackrel{63}{\underline{6}}$ pointed out that the $\mathrm{EPC}$ in the system is intrinsically weak, but can be enhanced strongly by magnetism ${ }^{64}$. Although EPC is still not enough to explain the high critical temperature, it is strong enough to have a non-negligible effect on SC. On the other hand, in the similar system of nickel pnictide superconductors, it seems the EPC plays the main role ${ }^{62}$. One may connect nickel pnictide superconductors with $\mathrm{MgCNi}_{3}$ by the similar EPC mechanism. The potential supeconductivity in doped $\mathrm{A}^{4+} \mathrm{CFe}_{3}$ may also help to figure out the superconducting mechanism, which needs to be futher confirmed in experimental and theoretical works. 


\section{CONCLUTIONS}

In this work, we theoretically investigated the electronic structure, magnetic properties, and lattice dynamics of the $3 d$ transition-metal based antiperovskite compounds $\mathrm{AXM}_{3}$. Based on the analysis of the doping effect in $\mathrm{AXM}_{3}$, we drew the magnetic phase diagram of $\mathrm{ACM}_{3}$. We suggested that superconducting antiperovskites can be found in the NM area but very close to FM boundary. In order to prove the deduction, we investigated a series of Cr-based antiperovskite $\mathrm{AXCr}_{3}(\mathrm{X}=\mathrm{C}, \mathrm{N})$. The results indicate that $\mathrm{AlCCr}_{3}, \mathrm{GaCCr}_{3}$, and $\mathrm{ZnNCr}_{3}$ are of $\mathrm{NM}$ ground state but very close to FM instability. Due to the large $N\left(E_{F}\right)$, these compounds have sizeable EPC. We calculated the phonon spectra and $\mathrm{EPC}$ of $\mathrm{MgCNi}_{3}$, $\mathrm{AlCCr}_{3}, \mathrm{GaCCr}_{3}$, and $\mathrm{ZnNCr}_{3}$. Our results confirm the strong $\mathrm{EPC}$ in $\mathrm{MgCNi}_{3}$, and show that $\mathrm{AlCCr}_{3}, \mathrm{GaCCr}_{3}$, and $\mathrm{ZnNCr}_{3}$ are moderate coupling BCS superconductors. Such compounds may be good candidates to figure out the role of SF. Moreover, some potential new antiperovskite Co-based and Fe-based superconductors are suggested.
$\mathrm{Up}$ to date, there is no definite evidence of $\mathrm{SF}$ in $\mathrm{MgCNi}_{3}$ single crystals ${ }^{20}$. Since such single crystals are not stoichiometric, the interplay between SC and magnetism are still puzzling. Our phase diagram and predictions could offer new route to figure out the issue. In experiments one can try to prepare such potential antiperovskite superconductors we predicted. Furthermore, by doping we can control the physical properties and may observe the magnetic-superconducting transition through quantum critical point. We hope our phase diagram and predictions can help to explore more $3 d$ transition-metal based antiperovskite superconductors and figure out the relation between SC and magnetism in the system.

\section{ACKNOWLEDGMENTS}

This work was supported by the National Key Basic Research under Contract No. 2011CBA00111, and the National Nature Science Foundation of China under Contract Nos. 51171177, 11304320, 11274311, 11174295, and U1232139. The calculations were partially performed at the Center for Computational Science, CASHIPS.
* wjlu@issp.ac.cn

† ypsun@issp.ac.cn

1 Onnes H K 1911 Comm. Phys. Lab. Univ. Leiden 122124

2 Cooper L N 1956 Phys. Rev. 1041189

3 Bardeen J, Cooper L N, and Schrieffer J R 1957 Phys. Rev. 106162

4 Bardeen J, Cooper L N, and Schrieffer J R 1957 Phys. Rev. 1081175

5 Bednorz J G and Müller K A 1986 Z. Phys. B: Condens. Matter 64189

6 Wu M K, Ashburn J R, Torng C J, Hor P H, and Meng R L 1987 Phys. Rev. Lett. 58908

7 Shimizu K, Kimura T, Furomoto S, Takeda K, Kontani K, Onuki Y, and Amaya K 2001 Nature 412316

8 Kamihara Y, Watanabe T, Hirano M, and Hosono H 2008 J. Am. Chem. Soc. 1303296

9 Maeno Y, Hashimoto H, Yoshida K, Nishizaki S, Fujita T, Bednorz J G, and Lichtenberg F 1994 Nature 372532

10 Takada K, Sakurai H, Takayama-Muromachi E, Izumi F, Dilanian R A, and Sasaki T 2003 Nature 42253

11 He T, Huang Q, Ramirez A P, Wang Y, Regan K A, Rogado N, Hayward M A, Haas M K, Slusky J S, Inumara K, Zandbergen H W, Ong N P, and Cava R J 2001 Nature 41154

12 Rosner H, Weht R, Johannes M D, Pickett W E, and Tosatti E 2001 Phys. Rev. Lett. 88, 027001

13 Singh D J and Mazin I I 2001 Phys. Rev. B 64 140507(R)

14 Shim J H, Kwon S K, and Min B I 2001 Phys. Rev. B 64 $180510(\mathrm{R})$

15 Mollah S 2004 J. Phys.: Condens. Matter 16 R1237

16 Lee H S Jang D J, Lee H G, Lee S I, Choi S M, and Kim C J 2007 Adv. Mater. 191807

17 Gordon R T, Zhigadlo N D, Weyeneth S, Katrych S, and
Prozorov R 2013 Phys. Rev. B 87094520

18 Sieberer M, Mohn P, and Redinger J 2007 Phys. Rev. B 75024431

19 Shao D F, Lu W J, Lin J C, Tong P, Jian H B, and Sun Y P 2013 J. Appl. Phys. 113023905

20 Lee H S, Jang D J, Lee H G, Kang W, Cho M H, and Lee S I 2008 J. Phys.: Condens. Matter 20, 255222

21 Pribulová Z, Kačmarčík J, Marcenat C, Szabó P, Klein T, Demuer A, Rodiere P, Jang D J, Lee H S, Lee S I, and Samuely P 2011 Phys. Rev. B 83104511

22 Diener P, Rodière P, Klein T, Marcenat C, Kacmarck J, Pribulova Z, Jang D J, Lee H S, Lee H G, and Lee S I 2009 Phys. Rev. B $\mathbf{7 9} 220508(\mathrm{R})$

23 Jang D J, Lee H S, Lee H G, Cho M H, and Lee S I 2009 Phys. Rev. Lett. 103047003

24 Hong H, Upton M, Said A H, Lee H S, Jang D J, Lee S I, Xu R, and Chiang T C 2010 Phys. Rev. B 82134535 (2010).

25 Jha P K, Gupta S D, and Gupta S K 2012 AIP Advances 2022120

26 Tong $\mathrm{P}$ and Sun Y P 2012 Adv. Cond. Matter Phys. 2012 903239

27 Loison C, Leithe-Jasper A, and Rosner H 2007 Phys. Rev. $B 75205135$

28 Fruchart D, Fruchart R, L'heritier P, Kanematsu K, Madar R, Misawa S, Nakamura Y, Webster P J, and Ziebeck K R A 1988 Alloys and Compounds of D-Elements With Main Group Elements (Berlin: Springer)

29 Uehara M, Yamazaki T, Kôri T, Kashida T, Kimishima Y, and Hase I 2007 J. Phys. Soc. Jpn. 76034714

30 Uehara M, Uehara A, Kozawa K, Yamazaki T, and Kimishima Y 2010 Physica C 470S688

31 Tong P, Wang B S, and Sun Y P 2013 Chin. Phys. B 22 
067501

32 Wiendlocha B, Tobola J, Kaprzyk S, Fruchart D, and Marcus J 2006 Phys. Status Solidi B 243351

33 Wiendlocha B, Tobola J, and Kaprzyk S 2006 Phys. Rev. B 73134522

${ }^{34}$ Wiendlocha B, Tobola J, Kaprzyk S, and Fruchart D 2007 J. Alloys Compd. 442289

35 Tütüncü H M and Srivastava G P 2012 J. Appl. Phys. 112 093914

36 Tütüncü H M and Srivastava G P 2013 J. Appl. Phys. 114 053905

37 Blöchl P E 1994 Phys. Rev. B 5017953

38 Torrent M, Jollet F, Bottin F, Zérah G, and Gonze X 2008 Comp. Mater. Sci. 42337

${ }^{39}$ Gonze X, Beuken J M, Caracas R, Detraux F, Fuchs M, Rignanese G M, Sindic L, Verstraete M, Zerah G, Jollet F, et al. 2002 Comp. Mater. Sci. 25478

40 Gonze X, Amadon B, Anglade P M, Beuken J M, Bottin F, Boulanger P, Bruneval F, Caliste D, Caracas R, Cote M, et al. 2009 Comput. Phys. Comm. 1802582

${ }^{41}$ Gonze X, Rignanese G M, Verstraete M, Beuken J M, Pouillon Y, Caracas R, Jollet F, Torrent M, Zerah G, Mikami M, et al. 2005 Z. Kristallogr. 220558

42 Monkhorst H J and Pack J D 1976 Phys. Rev. B 135188

43 Baroni S, de Gironcoli S, Dal Corso A, and Giannozzi P 2001 Rev. Mod. Phys. 73515

44 Vanderbilt D 1990 Phys. Rev. B 417892

45 Giannozzi P, et al. 2009 J. Phys.: Condens. Matter 21 395502

46 Perdew J P, Burke K, and Ernzerhof M 1996 Phys. Rev. Lett. 773865

47 Shao D F, Lu W J, Lin S, Tong P, and Sun Y P $2013 A d v$.
Condens. Matter. Phys. 2013136274

48 Ignatov A Y, Savrasov S Y, and Tyson T A 2003 Phys. Rev. B 68 220504(R)

49 Heid R, Renker B, Schober H, Adelmann P, Ernst D, and Bohnen K P 2004 Phys. Rev. B 69092511

50 Jha P K 2005 Phys. Rev. B 72214502

51 Tütüncü H M and Srivastava G P 2006 J. Phys.: Condens. Matter 1811089

52 Wälte A, Fuchs G, Müller K H, Handstein A, Nenkov K, Narozhnyi V N, Drechsler S L, Shulga S, Schultz L, and Rosner H 2004 Phys. Rev. B $\mathbf{7 0} 174503$

53 Dolgov O V, Golubov A A, Mazin I I and Maksimov E G 2008 J. Phys.: Condens. Matter 20434226

54 McMillan W L 1968 Phys. Rev. 167331

55 Dynes R 1972 Solid State Commun. 10615

56 Allen P B and Dynes R C 1975 Phys. Rev. B 12905

57 Ortenzi L, Biermann S, Andersen O K, Mazin I I , and Boeri L 2011 Phys. Rev. B 83 100505(R)

58 Schaak R E, Avdeev M, Lee W L, Lawes G, Zandbergen H W, Jorgensen J D, Ong N P, Ramirez A P, Cava R J 2004 J. Solid State Chem. 1771244

59 Mazin I I, Papaconstantopoulos D A, and Mehl M J 2002 Phys. Rev. B 65 100511(R)

60 Bose S K, Dolgov O V, Kortus J, Jepsen O, and Andersen O K 2003 Phys. Rev. B 67214518

61 Boeri L, Dolgov O V, and Golubov A A 2008 Phys. Rev. Lett. 101026403

62 Boeri L, Dolgov O V, and Golubov A A 2009 Physica C 469628

63 Mazin I I, Singh D J, Johannes M D, and Du M H 2008 Phys. Rev. Lett. 101057003

64 Boeri L, Calandra M, Mazin I I, Dolgov O V, and Mauri F 2010 Phys. Rev. B 82 020506(R) 